\title{
Adrenomedullin increases cardiac sympathetic nerve activity in normal conscious sheep
}

\author{
C J Charles, D L Jardine, M G Nicholls ${ }^{\mathbf{1}}$ and A M Richards
}

Christchurch Cardioendocrine Research Group, Christchurch School of Medicine and Health Sciences, PO Box 4345, Christchurch, New Zealand

${ }^{1}$ Department of Internal Medicine, United Arab Emirates University, Al Ain, United Arab Emirates

(Requests for offprints should be addressed to C J Charles; Email: chris.charles@chmeds.ac.nz)

\begin{abstract}
The sympathetic nervous system and adrenomedullin (AM) both participate in the regulation of cardiac and circulatory function but their interaction remains uncertain. We have examined the effects of AM on cardiac sympathetic nerve activity (CSNA) and hemodynamics and contrasted these effects with pressure-matched nitroprusside (NP) administration in normal conscious sheep. Compared with vehicle control, arterial pressure fell similarly with AM $(P=0 \cdot 04)$ and NP $(P<0 \cdot 001)$. Heart rate rose in response to both AM $(P<0 \cdot 001)$ and NP $(P=0.002)$ but the rise with AM was significantly greater than that induced by NP $(P<0 \cdot 001)$. Cardiac output increased in response to AM compared with both control
\end{abstract}

and NP (both $P<0 \cdot 001)$. CSNA burst frequency (bursts/ $\mathrm{min})$ were increased in response to both AM $(P<0 \cdot 001)$ and NP $(P=0.005)$ with the rise in burst frequency being greater with AM compared with NP $(P<0 \cdot 001)$. CSNA burst area/min was also raised by both $\operatorname{AM}(P=0.03)$ and NP $(P=0.002)$ with a trend for burst area being greater with AM than NP $(P=0 \cdot 07)$. CSNA burst incidence (bursts/100 beats) showed no significant differences between any treatment day. In conclusion, we have demonstrated that AM is associated with a greater increase in CSNA and heart rate for a given change in arterial pressure than seen with the classic balanced vasodilator NP.

Journal of Endocrinology (2005) 187, 275-281

\section{Introduction}

Sympathetic nervous activity (SNA) is a pivotal element of normal cardiac and circulatory regulation. Adrenomedullin (AM) is a 52 amino acid peptide also involved in the regulation of vascular tone and pressure/volume homeostasis (Samson 1999). Both entities show enhanced activation in cardiac injury and in heart failure, but the interaction between AM and the sympathetic nervous system is uncertain. Hemodynamic actions of infused AM include powerful and sustained reductions in arterial pressure in sheep (Parkes \& May 1995, Charles et al. 1997) and humans (Lainchbury et al. 1997, 2000). This is associated with rises in cardiac output, falls in peripheral resistance and increases in heart rate (Parkes \& May 1995, Charles et al. 1997). During infusions of AM, plasma norepinephrine may be decreased as in normal sheep (Charles et al. 1997), possibly unchanged as in some human studies (Lainchbury et al. 1997) or increased as in other human work (Lainchbury et al. 2000, Troughton et al. 2000, McGregor et al. 2001). It is unclear whether $\mathrm{AM}$-induced increases in heart rate are purely baroreceptor mediated or whether in fact the associated changes in SNA are re-set by AM compared with responses to comparable hypotension induced by other vasodilators.
There is also the possibility that AM may directly activate central nervous system sympathetic output. Increases in heart rate with AM do not appear to be solely mediated by baroreflexes as tachycardia persists beyond hypotension in sheep infused with AM (Parkes \& May 1995). A number of studies have reported regional SNA responses to AM. In anesthetized rats AM increases abdominal sympathetic discharge (Takahashi et al. 1994). However, whether this augmented SNA is solely baroreflex mediated or not remains contentious as AM-induced rises in renal SNA are reduced compared with nitroprusside (NP) in the face of comparable reduction in blood pressure in rabbits (Fukuhara et al. 1995) but increased compared with NP effects in rats (Saita et al. 1998). Sympathetic activation through administration of different endogenous and exogenous vasodilators or through the onset of cardiac injury or heart failure can show great regional selectivity (Ninomiya et al. 1971, Pagani et al. 1974), which highlights the need to measure SNA efferent traffic to the organ of interest. Our recent establishment of a model in which it is possible to obtain reliable direct recordings of cardiac efferent SNA (CSNA) in conscious sheep (Jardine et al. 2002) has placed us in a unique position to study the effects of AM on CSNA. Accordingly, we have examined the effects of AM on CSNA and hemodynamics and 
contrasted these effects with pressure-matched NP administration in normal conscious sheep.

\section{Materials and Methods}

The Animal Ethics Committee of the Christchurch School of Medicine and Health Sciences approved the study protocol. Eight Coopworth ewes (Lincoln University Farm, Christchurch, New Zealand) were housed in an air-conditioned light-controlled room and received a diet of lucerne chaff and food pellets providing $75 \mathrm{mmol}$ sodium and $150 \mathrm{mmol}$ potassium per day. Under general anesthesia (induced by $17 \mathrm{mg} / \mathrm{kg}$ thiopentone sodium and maintained by a mixture of halothane, nitrous oxide and oxygen) a left lateral thoracotomy was performed to allow up to five stainless steel needle electrodes to be inserted and glued in the thoracic cardiac nerves as previously described (Jardine et al. 2002). Connecting leads were sutured to the mediastinum and exteriorized dorsally through the chest wall. During the same anesthetic, via a $5 \mathrm{~cm}$ neck incision, a carotid artery was cannulated (16 G Angiocath; Becton Dickinson, Sandy, UT, USA) for direct measurement of arterial pressure and heart rate, polyethylene catheters were placed in the jugular veins for blood sampling and measurement of right atrial pressure and a Swan-Ganz thermodilution catheter (Edwards Lifesciences, Irvine, CA, USA) was placed in the pulmonary artery via the jugular vein for measurements of cardiac output. The animals were allowed to recover for at least 4 days before experiments.

CSNA recordings were made from pairs of electrodes via a preamplifier with an active probe (DAM-80; World Precision Instruments, Sarasota, FL, USA). The raw signal was amplified $(\times 1000)$, filtered between 300 and 3000 $\mathrm{Hz}$ and integrated using a time constant of $100 \mathrm{~ms}$. The integrated nerve signal was digitally converted using in-house software (sampling rate $200 \mathrm{~Hz}$ ) and postganglionic efferent sympathetic activity was identified in all animals by the following characteristics: (i) bursts were synchronized to the diastolic phase of the arterial pulse; (ii) bursts decreased during sympathetic blockade with hexamethonium infusion $(2 \mathrm{mg} / \mathrm{kg}$ over $2 \mathrm{~h})$ on day 3 after thoracotomy; and (iii) there was an inverse relationship between burst area and diastolic blood pressure during baroreflex tests undertaken on each recording day. Only recordings with a signal-to-noise ratio of greater than 2 were analyzed. Using these criteria on the first day, the best signal from all possible electrode combinations was selected and used for subsequent recordings. CSNA was quantified by: (i) counting the number of bursts per minute (burst frequency); (ii) counting the number of bursts per 100 heart beats (burst incidence); and (iii) measuring the area under the integrated signal per minute (burst area/min) and burst area/100 beats.

Each animal was studied on three occasions receiving vehicle (haemaccel) control, AM and NP using a study design similar to our previous study (Charles et al. 2001). AM was infused i.v. at a dose of $5.5 \mathrm{pmol} / \mathrm{kg}$ per min (33 ng/kg per min for $120 \mathrm{~min}$ ) in a total volume of $40 \mathrm{ml}$ haemaccel and NP was titrated (dose range $2.5-20 \mathrm{mg} / \mathrm{h}$ ) to achieve a fall in mean arterial pressure (MAP) matched to that induced by AM. Control and AM were administered in a balanced random order design whilst NP always followed AM to allow a matched fall in MAP. Human AM-52 was synthesized as previously described (Charles et al. 1997).

Arterial pressure recordings, using an in-house online data acquisition system, commenced $30 \mathrm{~min}$ before infusions and were continued for $60 \mathrm{~min}$ after infusion. Heart rate and pressures were digitally integrated in $5 \mathrm{~min}$ recording periods and data recorded at preset intervals throughout the study. Cardiac output (thermodilution) was measured in triplicate (three values within 10\%) at preset intervals for the duration of infusions. Calculated total peripheral resistance (CTPR) was calculated as MAP divided by cardiac output. Venous blood was drawn at preset intervals during the study protocol. Blood was taken into chilled EDTA tubes, centrifuged and the plasma stored at $-80{ }^{\circ} \mathrm{C}$ before assay for AM (Lewis et al. 1998).

\section{Statistics}

Results are expressed as means \pm S.E.M. Two-way ANOVA with time as a repeated measure was used to determine time and treatment differences between AM, $\mathrm{NP}$ and control arms of the study. Statistical significance was assumed at $P<0 \cdot 05$.

\section{Results}

Experiments were completed without mishap and data collection was complete. There was no significant difference in baseline levels of any variables between study days except in the case of MAP where levels were virtually identical on the control and AM study days $(91.6 \pm 3.2$ vs $91.6 \pm 4.3 \mathrm{mmHg}$ respectively at time 0$)$ but were significantly lower on the NP day $(84.7 \pm 3.0 \mathrm{mmHg}$ at time $0, P=0.006$ baseline difference by ANOVA). Given this baseline difference, MAP results are expressed as change from baseline. CSNA burst area is an area under the curve measurement calculated in arbitrary units and as such these data are expressed as percentage change from baseline.

Compared with the time-matched control and in accord with study design, MAP fell similarly in response to AM $(P=0 \cdot 04)$ and NP $(P<0 \cdot 001)$ (Fig. 1). Although the fall in MAP was matched at the end of infusion $(-9 \cdot 6 \pm 1 \cdot 80$ and $-9.6 \pm 1.19 \mathrm{mmHg}$ at $120 \mathrm{~min}$ of $\mathrm{AM}$ and NP respectively), AM induced a slow onset depressor effect whereas pressures fell with NP more precipitously and, as such, the time-course of the MAP change was significantly different $(P=0 \cdot 011)$. Heart rate rose in response to both 


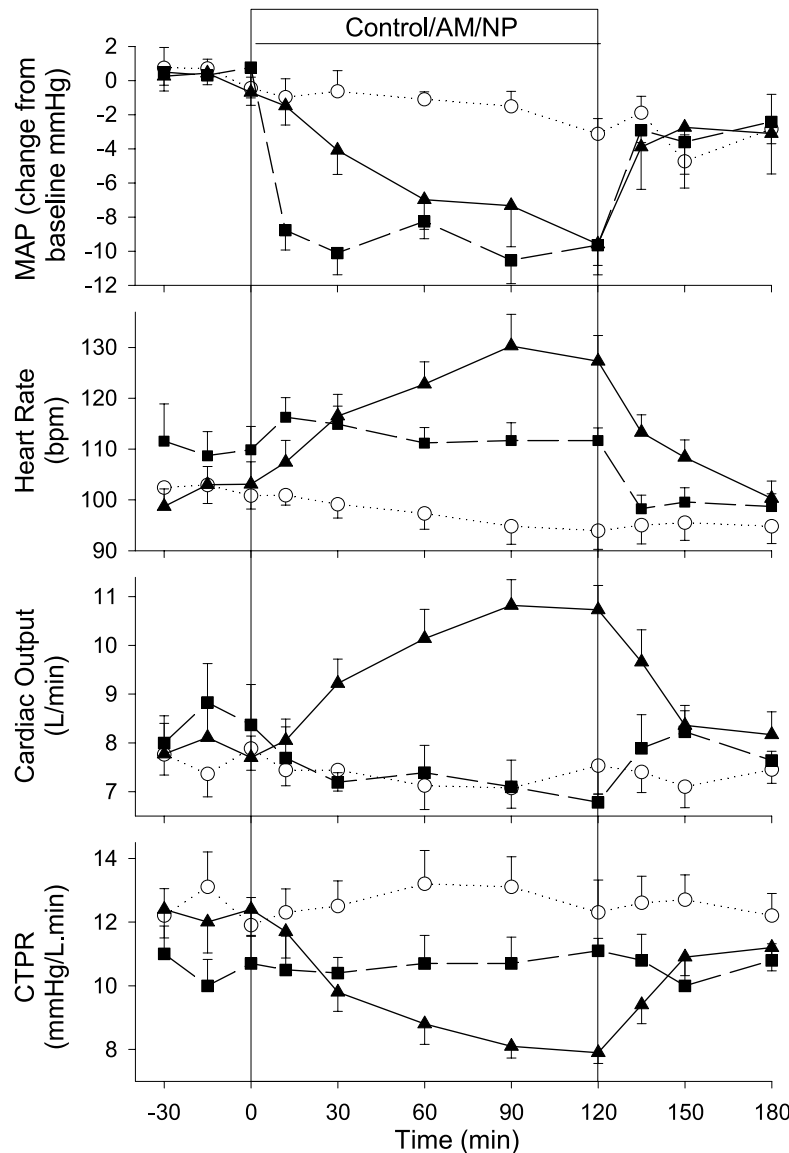

Figure 1 Mean arterial pressure (MAP), heart rate, cardiac output and calculated total peripheral resistance (CTPR) response to i.v. infusions of adrenomedullin (AM) at a dose of $5.5 \mathrm{pmol} / \mathrm{kg}$ per $\min (\boldsymbol{\Delta})$, nitroprusside (NP) titrated at a dose to match the fall in MAP ( $)$ or vehicle control $(O)$ in eight sheep. Values shown are means \pm S.E.M. Significant differences were observed for MAP-control vs AM $(P=0 \cdot 04)$, control vs NP $(P<0 \cdot 001)$, AM vs NP $(P=0.011)$; heart rate-control vs AM $(P<0 \cdot 001)$, control vs NP $(P<0.001)$, AM vs NP $(P=0.002)$; cardiac output-control vs AM $(P<0 \cdot 001)$, AM vs NP $(P<0 \cdot 001)$; and CTPR-control vs AM $(P<0 \cdot 001)$, AM vs NP $(P<0 \cdot 001)$.

AM $(P<0 \cdot 001)$ and NP $(P=0 \cdot 002)$ compared with control, but despite the more deliberate fall in arterial pressure induced by $A M$ the increase in heart rate with AM was significantly greater than that induced by NP $(P<0 \cdot 001)$. Cardiac output increased by approximately $31 / \mathrm{min}$ in response to AM $(P<0 \cdot 001$ vs both control and NP) but was unaltered by NP. Compared with control, CTPR was reduced in response to AM $(P<0.001$ vs both control and NP) but was unaltered by NP.

Figure 2 depicts original arterial pressure, CSNA and ECG recordings from a representative animal. Note that despite arterial pressure falling similarly at $120 \mathrm{~min}$ in response to AM and NP, heart rate response and CSNA were exaggerated during infusion of AM. As seen in
Fig. 3, CSNA burst frequency (burst/min) was increased in response to both AM $(P<0.001)$ and NP $(P=0.005)$ compared with control, with the rise in burst frequency induced by AM being greater than that induced by NP $(P<0 \cdot 001)$. CSNA burst area/min was also raised by both AM $(P=0.03)$ and NP $(P=0.002)$ compared with control. There was a trend for a different response between AM and NP $(P=0 \cdot 07)$ with burst area being greater with AM during the latter half of the infusions. CSNA burst incidence (bursts/100 beats) showed no significant difference between any treatment day, remaining between 60 and 70 bursts/100 beats. Similarly, CSNA burst area/100 beats was not significantly different between the three study days (data not shown).

Plasma immunoreactive AM levels remained at or below the detection limit for the assay during vehicle control infusions and NP, but rose as expected during AM infusions $(P<0.001 \mathrm{vs}$ both $)$ to approximately $15 \mathrm{pmol} / 1$ by the end of infusion (Fig. 4).

\section{Discussion}

The effect of AM on sympathetic traffic to the heart has not been previously reported. Whilst numerous biological actions for AM have been reported, the basis for many of these effects remains unclear. In particular, it is uncertain whether or not the direct vasodilator action of the hormone underpins many of its other actions. Accordingly, we have examined the effects of AM on CSNA and hemodynamics and contrasted these effects with pressurematched NP administration in normal conscious sheep. AM and NP similarly reduced MAP in accord with study design. However, both heart rate and CSNA (burst frequency) responses were significantly greater with AM compared with NP. Cardiac output was increased with AM but not NP, therefore the fall in CTPR was greater with AM.

Consistent hemodynamic effects of AM have been reported by a number of authors. These actions include lowering of arterial pressure associated with increases in cardiac output and falls in peripheral resistance (Charles et al. 1997, Lainchbury et al. 2000, Troughton et al. 2000). We have previously reported the effects of AM compared with pressure-matched NP in another group of sheep undergoing similar experiments but without concurrent CSNA recordings (Charles et al. 2001). Less clear is whether baroreceptor-mediated increases in sympathetic activity (for a given change in blood pressure) are altered by AM compared with NP, a standard agent used for assessing baroreflex-modulated responses to vasodilation (Casadei \& Paterson 2000). As expected, blood pressure fell more rapidly with NP during the first hour of the infusion and so comparisons of CSNA and heart rate responses were more appropriate during the second hour when blood pressures were similar, that is approximately 


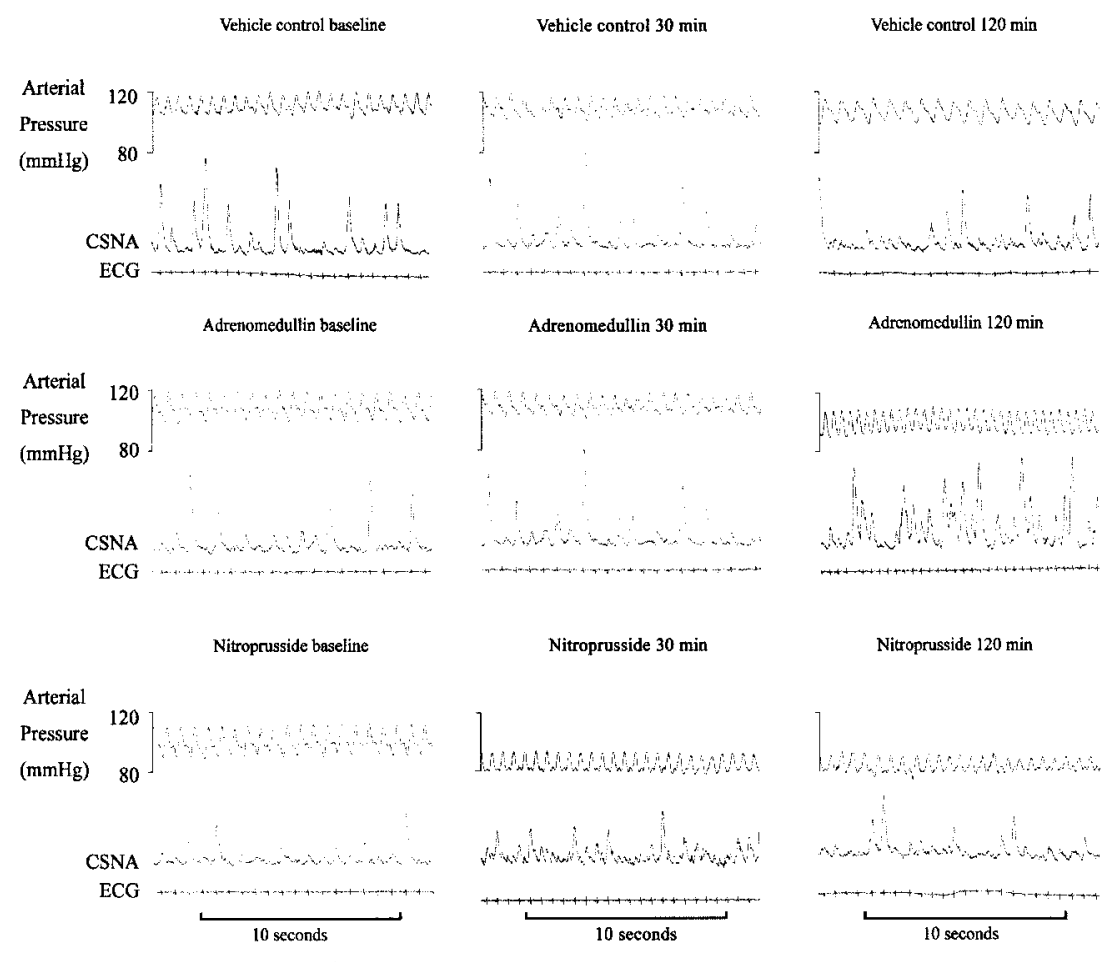

Figure 2 Sample tracing of arterial pressure, cardiac sympathetic nerve activity (CSNA) and ECG before (left), after 30 min (middle) and at end of (right) $2 \mathrm{~h}$ infusions of vehicle control (top), adrenomedullin (center) and nitroprusside (bottom panels).

$10 \mathrm{mmHg}$ below baseline. Both CSNA (burst frequency) and heart rate were higher during AM, suggesting that AM may exaggerate the baroreflex-modulated response to vasodilation.

Reports of AM's effects on sympathetic activity have demonstrated mixed results. Plasma norepinephrine responses to AM administration show mixed responses (Charles et al. 1997, Lainchbury et al. 1997, 2000, Troughton et al. 2000, McGregor et al. 2001). However, plasma catecholamines are a crude index of sympathetic activity and do not allow sympathetic activity to be measured to specific organs. An early study administering AM to rabbits showed that dose-dependent reduction in MAP was associated with concomitant increases in renal SNA (Fukuhara et al. 1995). However, the SNA responses were less than those induced by pressure-matched NP, suggesting that AM attenuates the reflex-mediated sympathetic response to hypotension. In contrast, studies in rats have shown that AM provoked an enhanced renal SNA response to hypotension compared with NP (Saita et al. 1998). Moreover, the renal SNA response to AM was suggested to be predominantly dependent on the arterial baroreceptor as sinoaortic denervation markedly attenuated the responses. It is important to note that there can be great selectivity of sympathetic reflex activity to different tissue beds (Ninomiya et al. 1971, Pagani et al. 1974), highlighting the need to measure SNA efferent traffic to the organ of interest. The present study is the first to report effects of AM on efferent SNA traffic directed to the heart. Given the pivotal role of sympathetic drive specifically to the heart in many cardiac disease settings, and the known increase in plasma AM after myocardial infarction and in heart failure, these findings may further clarify the links between humoral status and cardiac autonomic drive in health and disease. AM clearly induced activation of CSNA (as measured by burst frequency and trends in burst area/minute), which was significantly augmented compared with that induced by pressurematched NP. This suggests that efferent sympathetic traffic directed to the heart may have been augmented over baroreflex-mediated responses. However, it is important to note that CSNA burst incidence (bursts/100 beats) and burst area/100 beats (data not shown) were not significantly raised above either vehicle control or NP levels. CSNA bursts are entrained to heart rate (McAllen \& Malpas 1997), therefore one would expect burst frequency and burst area/minute to increase under conditions that increased heart rate. Thus, it remains unclear from the present study whether the primary effect of AM is to directly stimulate CSNA (via a central nervous system mechanism) resulting in increased heart rate, or to increase heart rate resulting in an increase in CSNA burst frequency. Future studies assessing the CSNA response to AM whilst heart rate is controlled (either by pacing or 


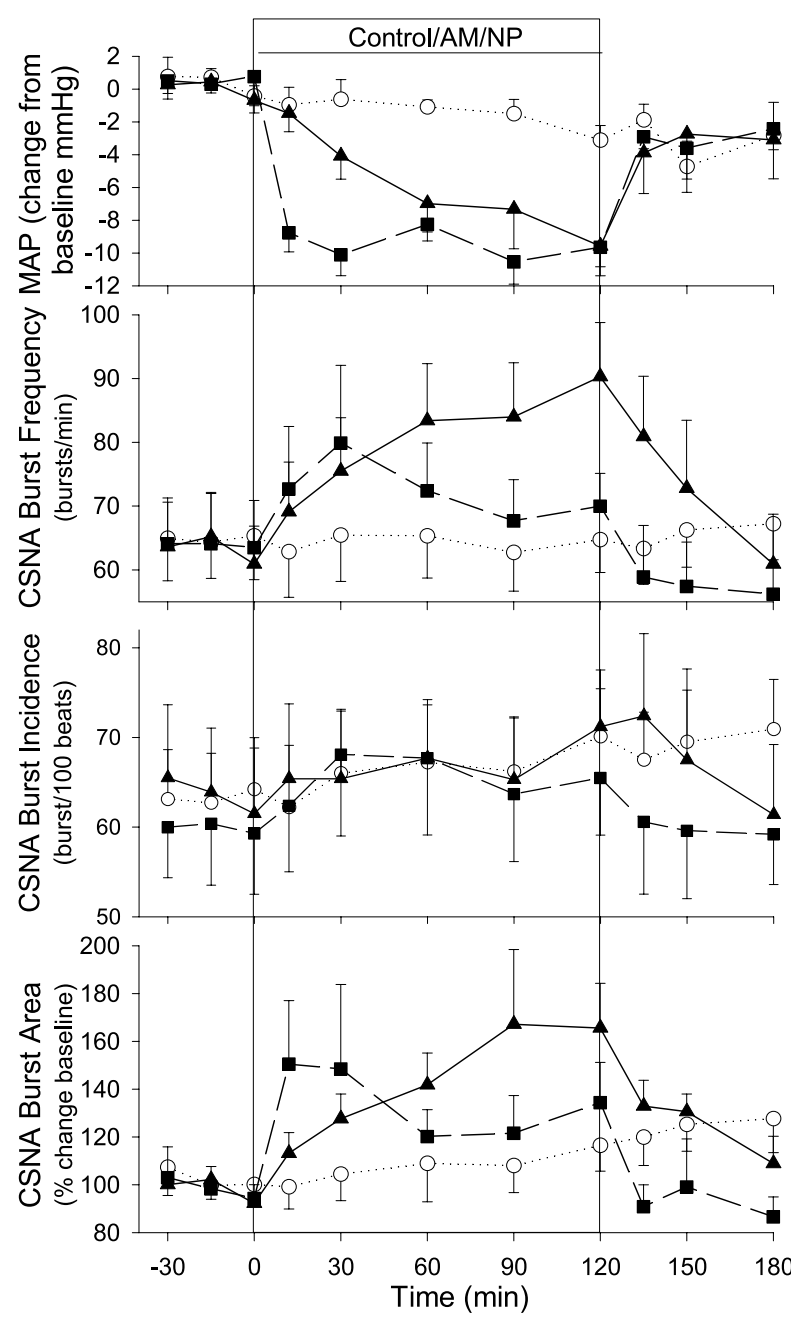

Figure 3 Mean arterial pressure (MAP) and cardiac sympathetic nerve activity (CSNA) response to i.v. infusions of adrenomedullin $(\mathrm{AM})$ at a dose of $5.5 \mathrm{pmol} / \mathrm{kg}$ per $\min (\boldsymbol{\Delta})$, nitroprusside (NP) titrated at a dose to match the fall in MAP (ם) or vehicle control $(\bigcirc)$ in eight sheep. Values shown are means \pm S.E.M. Significant differences were observed for MAP-control vs AM $(P=0.04)$, control vs NP $(P<0 \cdot 001)$, AM vs NP $(P=0.011)$; CSNA burst frequency-control vs AM $(P<0 \cdot 001)$, control vs NP $(P=0.005)$, AM vs NP $(P<0.001)$; CSNA burst area-control vs AM $(P=0.03)$, control vs NP $(P=0.002)$.

muscarinic blockers) should clarify this by eliminating cardiac entrainment of sympathetic bursts.

In considering the exaggerated rise in heart rate with AM compared with NP, it is important to note the pharmacodynamics of these agents are quite different. NP is a nitric oxide donor and therefore has a rapid onset and short duration of action, acts equally on all vascular beds, and probably does not cross the blood-brain barrier (Friederich \& Butterworth 1995). In contrast, AM acts via its own receptors, has a longer duration of action, effects only some vascular beds, and may have direct effects on

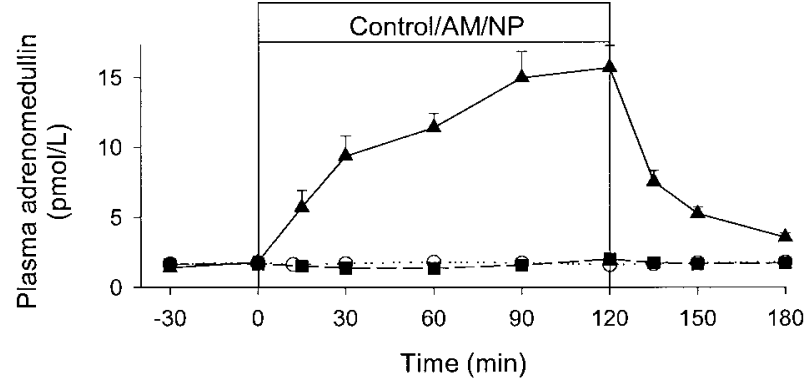

Figure 4 Plasma adrenomedullin (AM) response to i.v. infusions of $\mathrm{AM}$ at a dose of $5.5 \mathrm{pmol} / \mathrm{kg}$ per min $(\boldsymbol{\Lambda})$, nitroprusside (NP) titrated at a dose to match the fall in MAP ( $)$ or vehicle control $(\bigcirc)$ in eight sheep. Values shown are means \pm S.E.M. Significant differences were observed for plasma AM-control vs AM $(P<0 \cdot 001)$, AM vs NP $(P<0 \cdot 001)$.

the central nervous system (Samson 1999). Furthermore, NP is predominantly a venodilator and has no direct inotropic effect, both of which limit the cardiac output response to hypotension (Miletich \& Ivankovich 1978, Bauer \& Fung 1996). In contrast, AM is mainly an arterial vasodilator (Champion et al. 1997, Cockcroft et al. 1997) and may increase cardiac contractility (Parkes \& May 1995, Szokodi et al. 1998). The exaggerated heart rate response to AM observed in the present study may be due to increased sympathetic outflow from the central nervous system resulting in a greater rise in heart rate than that expected by baroreceptor activation alone. However, a complicating factor is that NP has been demonstrated to exert vagotonic activity on the sinus node (Chowdhary et al. 2002). Thus, an alternative explanation for the difference in heart rate response between AM and NP is that the heart rate response may be attenuated during NP-induced vasodilation. There is also some pharmacological evidence that AM may exert vagolytic effects via a central mechanism resulting in exaggeration of the heart rate response (Parkes \& May 1997). Indeed, Parkes \& May (1997) showed that AM-induced increases in heart rate were abolished during treatment with hexamethonium (but not sympathetic blockade). Clearly further studies examining both sympathetic and vagal outflow concurrently or utilizing vagal blockade of the heart are required to definitively address the underlying mechanisms.

AM's effect on cardiac output are likely to be multifactorial and could result from augmentation of efferent CSNA, alterations in cardiac preload or afterload, a direct positive inotropic action (Parkes \& May 1995, Szokodi et al. 1998) or via changes in heart rate. The present study demonstrates a substantial increase in cardiac output with AM quite distinct from NP, which had no observable effect. Accordingly, CTPR fell more for a given fall in arterial pressure with AM than NP. The available evidence points to AM being a potent vasodilator (Champion et al. 1997, Cockcroft et al. 1997). A likely explanation for cardiac output being increased more with AM is that, 
compared with NP, AM has less venodilatory action. Therefore, venous return (and stroke volume) is likely to be maintained during any heart rate increase, resulting in a proportionate increase in cardiac output. AM has been suggested to increase left ventricular contractility but we did not measure any indices for this and so cannot comment. Thus, it is likely that the AM-induced increase in cardiac output (secondary to increased CSNA and heart rate) mitigates falls in blood pressure despite large falls in peripheral vascular resistance.

Plasma concentrations of AM in some conditions are sufficient to suggest a role for the circulating peptide in volume and pressure homeostasis. Nonetheless, the precise role of AM in normal mammalian homeostasis is far from settled despite a growing body of literature. Proof that AM is a physiologically relevant hormone requires (among other things) the demonstration of end-organ responses to changes in plasma peptide levels encompassing those observed in normal health. At the dose employed in the present study, achieved plasma levels of approximately $15 \mathrm{pmol} / \mathrm{l}$ are just above the upper limit of physiological for humans (normal range in our laboratory with this assay is $2 \cdot 7-10 \cdot 1 \mathrm{pmol} / \mathrm{l})$. We have reported here significant augmentation of CSNA along with previously reported hemodynamic actions (compared with pressure-matched NP infusions) of AM infused at a dose which raises circulating levels just beyond the physiological range observed in humans. Although caution should be taken in comparing values across species, measurements of CSNA cannot be made in humans. Taken together, these results point to a physiological or pathophysiological role for circulating AM in pressure and volume homeostasis.

In the present study, animals were allowed to recover for at least 4 days before experiments commenced. This is a shorter recovery time than usually employed after thoracotomy in our laboratory (1-2 weeks). However, such a time-frame was necessary to utilize the available window during which nerve fields remain measurable, namely 7-10 days (Jardine et al. 2002). In that study, following implantation of CSNA recording electrodes, arterial pressure, heart rate and CSNA indices are raised initially but stable from day $4 / 5$ after operation onwards (Jardine et al. 2002). We have also previously demonstrated that a wide range of neurohumoral indices including plasma catecholamines, plasma renin activity and aldosterone return to pre-surgical levels within $24 \mathrm{~h}$ of sham-infarction thoracotomy (Rademaker et al. 2000). Furthermore, there was no baseline difference between most indices measured between experimental days and all of these indices were within the normal physiological range for sheep in our laboratory. Taken together, we believe the time-frame used for the present study was appropriate for allowing suitable recovery after surgery and completing studies with measurable nerve fields.

In conclusion, CSNA and circulating AM both participate in regulation of cardiac and vascular function during health and in disease states such as heart failure. Their interaction is incompletely understood. In the current experiment we have measured for the first time CSNA response to AM administration and demonstrated that $\mathrm{AM}$ is associated with a greater increase in CSNA and heart rate for a given change in arterial pressure than seen with the classic balanced vasodilator NP. It remains unclear whether the primary effect of AM is to directly stimulate CSNA (via a central nervous system mechanism) or to increase heart rate (possibly via vagal withdrawal). Future studies assessing the CSNA response to AM whilst heart rate is controlled or measuring CSNA and vagal activity concurrently are required to clarify underlying mechanisms.

\section{Acknowledgements}

We are grateful to staff of the Christchurch School of Medicine and Health Sciences Animal Laboratory for assistance with animal studies and Christchurch Cardioendocrine Laboratory staff for hormone assays. The authors declare that there is no conflict of interest that would prejudice the impartiality of this scientific work.

\section{Funding}

Support was provided through grants from the National Heart Foundation of New Zealand, Lotteries Heath Research and Health Research Council of New Zealand.

\section{References}

Bauer JA \& Fung HL 1996 Arterial versus venous metabolism of nitroglycerin to nitric oxide: a possible explanation of organic nitrate venoselectivity. Journal of Cardiovascular Pharmacology 28 371-374.

Casadei B \& Paterson DJ 2000 Should we use nitrovasodilators to test baroreflex sensitivity? Journal of Hypertension 18 3-6.

Champion HC, Lambert DG, McWilliams SM, Shah MK, Murphy WA, Coy DH \& Kadowitz PJ 1997 Comparison of responses to rat and human adrenomedullin in the hindlimb vascular bed of the cat. Regulatory Peptides 70 161-165.

Charles CJ, Rademaker MT, Richards AM, Cooper GJS, Coy DH, Jing NY \& Nicholls MG 1997 Hemodynamic, hormonal, and renal effects of adrenomedullin in conscious sheep. American Journal of Physiology 272 R2040-R2047.

Charles CJ, Nicholls MG, Rademaker MT \& Richards AM 2001 Comparative actions of adrenomedullin and nitroprusside: interactions with ANG II and norepinephrine. American Journal of Physiology 281 R1887-R1894.

Chowdhary S, Nuttall SL, Coote JH \& Townend JN 2002 L-arginine augments cardiac vagal control in healthy human subjects. Hypertension 39 51-56.

Cockcroft JR, Noon JP, Gardner-Medwin J \& Bennett T 1997 Haemodynamic effects of adrenomedullin in human resistance and capacitance vessels. British Journal of Clinical Pharmacology 44 57-60. 
Friederich JA \& Butterworth JF 1995 Sodium nitroprusside: twenty years and counting. Anesthesia and Analgesia 81 152-162.

Fukuhara M, Tsuchihashi T, Abe I \& Fujishima M 1995 Cardiovascular and neurohormonal effects of intravenous adrenomedullin in conscious rabbits. American Journal of Physiology 269 R1289-R1293.

Jardine DL, Charles CJ, Melton IC, May CN, Forrester MDE, Bennett SI \& Ikram H 2002 Continual recording of cardiac sympathetic nerve activity in conscious sheep. American Journal of Physiology 282 H93-H99.

Lainchbury JG, Cooper GJS, Coy DH, Jiang NY, Lewis LK, Yandle TG, Richards AM \& Nicholls MG 1997 Adrenomedullin: a hypotensive hormone in man. Clinical Science 92 467-472.

Lainchbury JG, Troughton RW, Lewis LK, Yandle TG, Richards AM \& Nicholls MG 2000 Hemodynamic, hormonal and renal effects of short-term adrenomedullin infusion in healthy volunteers. Journal of Clinical Endocrinology and Metabolism $\mathbf{8 5}$ 1016-1020.

Lewis LK, Smith MW, Yandle TG, Richards AM \& Nicholls MG 1998 Adrenomedullin (1-52) measured in human plasma by radioimmunoassay: plasma concentration, adsorption and storage. Clinical Chemistry 44 571-577.

McAllen RM \& Malpas SC 1997 Sympathetic burst activity: characteristics and significance. Clinical and Experimental Pharmacology and Physiology 24 791-799.

McGregor DO, Troughton RW, Frampton C, Lynn KL, Yandle T, Richards AM \& Nicholls MG 2001 Hypotensive and natriuretic actions of adrenomedullin in subjects with chronic renal impairment. Hypertension 37 1279-1284.

Miletich DJ \& Ivankovich AD 1978 Sodium nitroprusside and cardiovascular hemodynamics. International Anesthesiology Clinics 16 31-49.

Ninomiya I, Nisimaru N \& Irisawa H 1971 Sympathetic nerve activity to spleen, kidney and heart in response to baroreceptor input. American Journal of Physiology 221 1346-1351.
Pagani M, Schwartz PJ, Banks R, Lombardi F \& Malliani A 1974 Reflex responses to sympathetic preganglionic neurones initiated by different cardiovascular receptors in spinal animals. Brain Research 68 215-225.

Parkes DG \& May CN 1995 ACTH-suppressive and vasodilator actions of adrenomedullin in conscious sheep. Journal of Neuroendocrinology 7 923-929.

Parkes DG \& May CN 1997 Direct cardiac and vascular actions of adrenomedullin in conscious sheep. British Journal of Pharmacology $1201179-1185$.

Rademaker MT, Cameron VA, Charles CJ, Espiner EA, Nicholls MG, Pemberton CJ \& Richards AM 2000 Neurohormones in an ovine model of compensated postinfarction left ventricular dysfunction. American Journal of Physiology 278 H731-H740.

Saita M, Ishizuka Y, Kato K, Hanamori T, Kitamura K, Eto T \& Kannan H 1998 Cardiovascular and sympathetic effects of proadrenomedullin NH2-terminal 20 peptide in conscious rats. Regulatory Peptides 77 47-53.

Samson WK 1999 Adrenomedullin and the control of fluid and electrolyte homeostasis. Annual Review of Physiology 61 363-389.

Szokodi I, Kunnunen P, Tavi P, Weckstrom M, Toth M \& Ruskoaho H 1998 Evidence for cAMP-independent mechanisms mediating the effects of adrenomedullin, a new inotropic peptide. Circulation 97 1062-1070.

Takahashi H, Watanabe TX, Nishimura M, Nakanishi T, Sakamoto M, Yoshimura Y, Komiyama T, Masuda M \& Murakami T 1994 Centrally induced vasopressor and sympathetic responses to a novel endogenous peptide, adrenomedullin, in anesthetized rats. American Journal of Hypertension 7 478-482.

Troughton RW, Lewis LK, Yandle TG, Richards AM \& Nicholls MG 2000 Hemodynamic, hormone and urinary effects of adrenomedullin infusion in essential hypertension. Hypertension 36 588-593.

Received in final form 18 August 2005 Accepted 23 August 2005 
